Ein Abhandenkommen im Sinne von $\S 935$ Abs. 1 BGB liegt nur vor, wenn der Eigentümer den Besitz an einer Sache ohne seinen Willen verliert (Palandt-Bassenge, § 935 Rz. 3 mwN). Die Klägerin hatte aber niemals unmittelbaren Besitz an den Gegenständen.

Im Übrigen hätten die Eheleute A das Eigentum auch nach § 937 BGB erworben, da sie die Gegenstände über 10 Jahre im Eigenbesitz hatten und nicht bösgläubig waren, wie bereits dargelegt wurde.
Es konnte deshalb auch offen bleiben, ob der Beklagte überhaupt noch im Besitz der Gefäße und Schalen ist. Insoweit hatte er schon in erster Instanz vorgetragen, die Gegenstände weiter veräußert zu haben.

Zutreffend hat das Landgericht auch den Klageantrag zu 2) abgewiesen. Auf die diesbezüglichen Ausführungen in den Entscheidungsgründen des angefochtenen Urteils wird Bezug genommen. (Entscheidung von der Redaktion bearbeitet.)
Da die Eheleute A Eigentum erworben und der Beklagte die Gegenstände käuflich erworben hat, hat er das Eigentum von den Berechtigten erworben, so dass es auf seine Gut- oder Bösgläubigkeit nicht ankommt.

\title{
Rückgabe von Raubkunst an die Republik Zypern
}

OLG München, Urteil vom 18. März 2013 - 19 U 4878/10

1. Die Republik Zypern hat ihr Eigentum an den streitgegenständlichen Kunstgegenständen nicht durch die Besetzung Nordzyperns durch die Türkische Republik Nordzypern verloren. Diese kann keine Rechtspersönlichkeit sein, weil sie von der Staatengemeinschaft nicht als völkerrechtliches Subjekt anerkannt wird.

2. Ansprüche auf Herausgabe beschlagnahmter Gegenstände sind nach Abschluss des Strafverfahrens auf dem Zivilrechtsweg geltend zu machen. (Leitsätze der Redaktion)

\section{Gründe:}

I.

- Die Klägerinnen begehren die Zustimmung des Beklagten zur Herausgabe von Ikonen, Kirchenkunst und frühgeschichtlichen Gegenständen, die die Staatsanwaltschaft München I im Rahmen des gegen den Beklagten wegen Hehlerei geführten Ermittlungsverfahrens 237 Js 230262/97 sichergestellt hat und die derzeit im Bayerischen Landeskriminalamt ... gelagert sind.

Die Klägerinnen berufen sich dabei auf ihr Eigentumsrecht an den genannten Gegenständen.

Sie tragen vor, dass sich die kirchlichen Gegenstände bis zur Besetzung des nördlichen Teil Zyperns durch türkische Truppen im Jahre 1974 dort befunden hätten. Sie seien in der Zeit danach aus den Kirchen, Klöstern und Museen im nördlichen Teil Zyperns entfernt und außer Landes gebracht worden. Die ebenfalls sichergestellten, aus Ausgrabungen stammenden prähistorischen Gegenstände, stünden aufgrund des geltenden Antiquities Law im Eigentum der Republik Zypern.

Der Beklagte ist der Auffassung, er bzw. seine Tochter oder seine Ehefrau seien Eigentümer der sichergestellten Gegenstände.
Auf Antrag der Staatsanwaltschaft München I hat der zuständige Strafsenat des Oberlandesgerichts München bereits am 3. Juni 2004 entschieden, dass einige der Gegenstände im Wege der Rechtshilfe an die Klägerinnen herauszugeben seien. Das Auswärtige Amt der Bundesrepublik Deutschland genehmigte die Rechtshilfe allerdings nicht und verwies die Klägerinnen auf den Zivilrechtsweg.

Aufgrund der daraufhin erhobenen Zivilklage hat das Landgericht München I mit der angefochtenen Entscheidung nach Durchführung der Beweisaufnahme der Klage in vollem Umfange stattgegeben.

In seiner Entscheidung stützt sich das Erstgericht zunächst darauf, dass die Kläger Eigentümer der asservierten Gegenstände seien und dem Beklagten kein Recht auf Herausgabe an ihnen zustehe. Es folgt aufgrund der durchgeführten Beweisaufnahme den Ausführungen des Sachverständigen Prof. D., wonach sich bestimmte Gegenstände bis 1974 in zypriotischen Kirchen, Klöstern und Kunstmuseen befunden hätten.

Auch soweit eine Zuordnung durch den Sachverständigen nicht eindeutig möglich gewesen sei, könnten die Klägerinnen die Zustimmung zur Herausgabe verlangen. Die Staatsanwaltschaft sei bereits dann zur Herausgabe an die Klägerinnen berechtigt, wenn feststünde, dass dem Beklagten keine Rechte an 
den Gegenständen zukämen. Ein Eigentumserwerb des Beklagten stünde nicht fest, da dem Beklagten von Anfang an der gute Glaube gefehlt habe.

Gegen dieses Urteil des Landgerichts München I richtet sich die Berufung des Beklagten, der damit seinen Antrag auf Klageabweisung aus erster Instanz weiter verfolgt. Der Beklagte beantragt in seiner Berufungsbegründung vom 07. Januar 2011 Aufhebung des Ersturteils und Klageabweisung, hilfsweise Zurückverweisung. Im ergänzenden Schriftsatz vom 06. Dezember 2012 wird der Zurückverweisungsantrag unbedingt gestellt. [...]

\section{II.}

Die zulässige Berufung des Beklagten erweist sich jedenfalls hinsichtlich der im Tenor aufgeführten Gegenstände als unbegründet.

Aufgrund der durchgeführten Beweisaufnahme ist das Erstgericht in nicht zu beanstandender Weise zur Überzeugung gelangt, dass die Gegenstände im Eigentum der Klägerinnen stehen und diese vom Beklagten die Zustimmung zur Herausgabe an sie verlangen können.

Hinsichtlich weiterer in der Klage aufgeführter Gegenstände vermag der Senat der Argumentation des Erstgerichts nicht zu folgen und hält eine weitere Beweisaufnahme für geboten.

Da aber hinsichtlich der im Tenor aufgeführten Gegenstände das Berufungsverfahren zur Endentscheidung reif ist, kann der Senat gemäß § 301 ZPO ein Teilurteil aussprechen. Die einzelnen Gegenstände sind hinreichend bestimmt und voneinander abgrenzbar. Es besteht auch nicht die Gefahr widersprechender Entscheidungen, da nicht einheitlich über den Anspruch auf Zustimmung zur Herausgabe hinsichtlich aller asservierten Gegenstände entschieden werden muss. [...]

Zutreffend weist die Berufung darauf hin, dass im landgerichtlichen Verfahren der Eigentumsnachweis für die Klägerinnen nicht hinsichtlich aller Gegenstände geführt sei. Dies beruht zum einen auf einer nicht vollständigen Beweisaufnahme des Erstgerichts, zum anderen auf einer Verkennung der Beweislast. Soweit das Erstgericht in seinem Urteil davon ausgeht, dass eine Herausgabe an die Kläger auch dann möglich ist, wenn deren Eigentum nicht nachgewiesen wird und stattdessen der Beklagte seine Rechte an den Gegenständen nachzuweisen hat, vermag der Senat dem nicht zu folgen. Dies würde zu einer Umkehr der Beweislast führen, die in den zivilprozessualen Grundsätzen keine Stütze hat. Nach der ungeschriebenen Grundregel des Zivilprozessrechts trägt der Anspruchsteller die Beweislast für die rechtsbegründenden, der Anspruchsgegner die für die rechtsvernichtenden, rechtshindernden und rechtshemmenden Tatbestandsmerkmale ( $B G H$ NJW 91, 1052). Da sich die Klagepartei bei ihrem Anspruch auf Zustimmung zur Herausgabe der asservierten Gegenstände auf ihr Eigentumsrecht stützt, ist sie grundsätzlich für dessen Nach- weis beweispflichtig. Etwas anderes ergibt sich auch nicht für die dem Rechtsstreit zugrundeliegende Besonderheit, dass die Gegenstände aufgrund eines Ermittlungsverfahrens nach § 94 StPO beschlagnahmt wurden. Zwar regelt bei nicht abgeschlossenen Verfahren $\S 111$ k StPO die Herausgabe, nach Abschluss des Straf- oder Ermittlungsverfahrens wie hier ist allerdings für einen Anspruch auf Herausgabe der Zivilrechtsweg eröffnet (vgl. OLG Frankfurt, 3 Ws 813/10; OLG Stuttgart, 2 Ws 165/01). Die Zivilgerichte entscheiden dann allein nach den Grundsätzen des Zivilprozessrechts, da sich das Verfahren grundsätzlich nach den Verfahrensvorschriften des angerufenen Gerichts richtet.

Unter diesem Gesichtspunkt stellt sich die Entscheidung des Erstgerichts jedenfalls hinsichtlich der im Tenor aufgeführten Gegenstände als zutreffend dar. Insoweit konnte das Landgericht aufgrund der durchgeführten Beweisaufnahme davon ausgehen, dass den Klägerinnen der Nachweis ihres Eigentums gelungen ist.

Die angeführten Gegenstände wurden sämtlich vom Sachverständigen Prof. Dr. D. örtlich und zeitlich bestimmten Museen, Kirchen und Klöstern in Nordzypern zugeordnet. Die Angriffe der Berufung sind nicht geeignet, Zweifel an der Richtigkeit der Überzeugungsbildung des Erstgerichts aufkommen zu lassen.

Soweit die Berufung die pauschale Bezugnahme des Erstgerichts auf die Ausführungen des Sachverständigen Prof. D. beanstandet, führt dies nicht zu einer anderen Beurteilung des Senates. Nach § 286 Abs. 1 S. 2 ZPO muss das erkennende Gericht die wesentlichen Grundlagen seiner Beweiswürdigung im Urteil zum Ausdruck bringen (BGH NJW 91, 1894). Dem kommt das Erstgericht in seinem Urteil nach, indem es seine Überzeugungsbildung zum schriftlichen Gutachten und der mündlichen Erläuterung von Prof. D. darlegt. Nicht erforderlich ist, dass darüber hinaus auf jeden Gegenstand gesondert eingegangen wird, sofern nicht die Umstände eine tiefergehende Würdigung erfordern. Der Berufungskläger versäumt es allerdings konkret aufzuzeigen, bei welchen Gegenständen die Bezugnahme auf das gesamte Gutachten nicht ausreichend gewesen wäre. Der Vorwurf, der Sachverständige habe die Gegenstände nur aufgrund von Lichtbildern zugeordnet, aber nicht in Augenschein genommen, vermag keine Zweifel an der Wertigkeit der Feststellungen des Sachverständigen zu begründen. Die Berufung kann nicht aufzeigen, welche anderen Erkenntnisse sich für den Sachverständigen bei einer Inaugenscheinnahme ergeben hätten. Im Übrigen gibt der Sachverständige in seiner Stellungnahme vom 24. März 2009 an, Gegenstände aus der Asservatensammlung des Bayerischen Landeskriminalamtes am 14. Oktober 1997 besichtigt und untersucht zu haben.

Einer näheren Betrachtung bedürfen Gutachten dann, wenn sie von anderen Gutachten abweichen, das Gericht selbst vom Gutachten abweichen will oder die Sachkunde und die Unvoreingenommenheit des Sachverständigen in Frage stehen. Hierzu ergeht sich die Berufung aber nur in haltlosen Vorwürfen des kollusiven Zusammenwirkens zwischen dem Sachverständigen und dem Vorsitzenden der Kammer des Erstgerichts. 
Ohne Rechtsfehler hat das Erstgericht auch den Schluss gezogen, dass sich bei der Mehrzahl der Ikonen die Herkunft aus Kirchen Nordzyperns auch durch die Methode der Stilbestimmung herleiten lässt. Der Sachverständige hat hierzu in seinem Gutachten darauf verwiesen, dass diese Methode in der Kunstgeschichte seit langem bewährt ist. Er hat im Folgenden die besonderen Merkmale einer Anzahl der sichergestellten Ikonen konkret benannt und sie aufgrund des ihnen ganz eigenen Stils als Gruppe den zypriotischen Ikonen zugewiesen. Dieser Expertise ist das Erstgericht gefolgt und hat das Eigentum der Klägerinnen insofern als erwiesen angesehen. [...]

Allerdings trägt das Gutachten nicht die vom Erstgericht vorgenommene Zuordnung der russischen Ikonen zu dieser Gruppe. Hier deutet bereits die Bezeichnung darauf hin, dass ihre Herkunft nicht zwingend Nordzypern ist. [...] Weitere Erklärungen zur Herkunft dieser Ikonen wurden von Seiten der Klägerinnen im Berufungsverfahren nicht abgegeben.

Die weiteren konkreten Beanstandungen zur Beweiswürdigung einzelner Gegenstände vermögen nicht zu überzeugen. So werden entgegen des Berufungsvorbringens die Gegenstände Ziffern I.2. (47), (64), (176), (194), (198) des Urteilstenors im Gutachten vom 24. September 2009, die Gegenstände Ziffern I.2. (144), (69) im Gutachten vom 30. August 2006 und die Gegenstände Ziffern I.2. (2), (28), (154), (175), (189), (196), (202) und (208) in beiden Gutachten vom Sachverständigen Prof. Dr. D. angeführt und Nordzypern zugeordnet.

Auch das Mosaik des HI. Thomas (Urteilsziffer I.2. (9)) wird im Gutachten vom 30. August 2006 eindeutig dem Apsismosaik der Kirche in Lythrankomi/Zypern zugeordnet. Hier wird lediglich die Asservatennummer einmal mit 137 und ein andermal mit 1.3.7. angegeben. Es besteht auch kein Zweifel, dass das Kunstwerk weiterhin vom BLKA asserviert wird. Die Klagepartei hat insofern unwidersprochen vorgetragen, dass es sich bei den restituierten Mosaikteilen um andere Gegenstände aus demselben Mosaik handelt.

Die Gegenstände befinden sich auch sämtlich noch in Verwahrung des B. Landeskriminalamtes. Wie sich aus der Mitteilung des BLKA vom 26. November 2009 (BI. 1237/1241) ergibt, wurden lediglich Asservate, die nicht Gegenstand dieses Rechtsstreites sind, an die zypriotischen Behörden herausgegeben.

Die Klägerinnen können sich auch auf ihre Eigentumsrechte berufen.

Die im Tenor genannten Gegenstände befanden sich bis zu ihrer Entfernung in Klöstern, Museen und Kirchen Nordzyperns. Dies steht fest auf Grund des Sachverständigengutachtens Prof. D. Mit der Herkunft der religiösen Kunstgegenstände und der Frage einer Zuordnung aufgrund des Kunststils setzt sich der Gutachter eingehend auseinander. Auf diese bezogen sich auch die Angaben des Beklagten und seines Verteidigers im strafrechtlichen Ermittlungsverfahren, die das Landgericht zu seiner Überzeugungsbildung heranzieht.

Die Klägerinnen haben ihre Eigentumsrechte an den genannten Gegenständen auch nicht wieder verloren. Insbesondere ist das Eigentum entgegen des Vorbringens der Berufung nach der Besetzung Nordzyperns im Jahre 1974 und Gründung einer Türkischen Republik Nordzypern (TRNZ) nicht auf diese übergegangen. Ein rechtsgeschäftlicher Eigentumserwerb wird nicht behauptet. Auch ein Eigentumserwerb im Rahmen einer Gesamtrechtsnachfolge kommt nicht in Frage, da die Türkische Republik Nordzypern nicht Gesamtrechtsnachfolger der Republik Zypern ist. Letztere besteht bekanntlich weiterhin. Regelungen der Türkischen Republik Nordzypern, die einen Eigentumsübergang auf sie belegen könnten, werden von der Berufung ebenfalls nicht vorgetragen. Letztlich kommt es darauf aber nicht an, da die Berufung nicht darlegen kann, dass die Türkische Republik Nordzyperns ein nach deutschem Recht rechtsfähiges Gebilde ist, das Eigentum an den Gegenständen erwerben könnte. Grundsätzlich haben ausländische Staaten, die in ihrer Eigenschaft als Fiskus in Deutschland am Rechtsverkehr teilnehmen, Rechtspersönlichkeit (vgl. Staudinger-Weick, Rn. 63 zu \& 21 BGB). In ähnlicher Weise kommt auch bestimmten Staatenvereinigungen innerstaatliche Rechtspersönlichkeit zu (Staudinger aaO). Voraussetzung ist jedoch jeweils, dass sie Völkerrechtssubjekte sind (Staudinger aaO). Die Türkische Republik Nordzypern wird allerdings von der Staatengemeinschaft nicht als völkerrechtliches Subjekt anerkannt. Die Türkei ist der einzige Staat, der die türkische Republik Nordzypern anerkennt.

Zwar übt die Republik Zypern seit der Besetzung des Nordens der Insel im Jahre 1974 durch die türkischen Streitkräfte keine Hoheit in diesem Territorium mehr aus, dennoch gehört das Gebiet de jure weiterhin zur Republik Zypern. Der UN-Sicherheitsrat erklärt die Proklamation der Türkischen Republik Nordzypern in seiner Resolution 541 für völkerrechtswidrig. Auch die Europäische Union geht von der Unteilbarkeit der Insel aus.

Soweit das Erstgericht davon ausgeht, dass weder der Beklagte noch seine Ehefrau und Tochter Eigentum an den Gegenständen begründen konnten, wird dies mit der Berufung nicht mehr gesondert angegriffen.

Die Herausgabeansprüche sind auch nicht verjährt.

Soweit die Berufung anführt, die Klage sei erst mehr als 30 Jahre nach der türkischen Invasion in Zypern gestellt worden und daher verjährt, übersieht sie, dass die Gegenstände frühestens und nicht spätestens mit der türkischen Invasion abhanden gekommen sind. Die Verjährungsfrist begann erst mit der Wegnahme der Gegenstände zu laufen, wobei dieser Zeitpunkt nicht genau bekannt ist, aber jedenfalls auch nach Auffassung der beklagten Partei nach dem Jahr 1974 liegt. (Entscheidung von der Redaktion bearbeitet.) 